\title{
Evaluating zero error noise thresholds by the replica method for Gallager code ensembles
}

\author{
Yoshiyuki Kabashima, Naoya Sazuka, \\ Kazutaka Nakamura \\ Dept. of Compt. Intel. \& Syst. Sci., \\ Tokyo Institute of Technology, \\ Yokohama 2268502, Japan
}

Abstract - The replica method, developed in statistical physics, is employed in conjunction with Gallager's methodology to accurately evaluate zero error noise thresholds for Gallager code ensembles. Our approach generally provides more optimistic evaluations than those reported in the information theory literature for sparse matrices; the difference vanishes as the parity check matrix becomes dense.

\section{INTRODUCTION}

Recent progress in the research of error correcting codes has revealed that Gallager's original code provides one of the best error correction performances to date $[1,2]$. A code of this type is defined by a randomly generated $N(1-R) \times N$ Boolean sparse parity check matrix $H$, composed of $j$ and $k$ non-zero (unit) elements per column and row, respectively. The code length and code rate are denoted by $N$ and $R$. The decoding error rate $P_{E}$ is a typical measure for the error correction ability of a given code. Since Gallager's codes are constructed randomly, an average error rate $\left[P_{E}\right]_{H}$ over a given ensemble, specified by the parameters $j$ and $k$, is introduced to characterize their typical performance [3]; where $[\cdots]_{H}$ represents an average over the code ensemble. For the maximum likelihood decoding and a binary symmetric channel (BSC), the average error rate is defined as $\left[P_{E}\right]_{H}=\left[\sum_{\boldsymbol{n}^{0}} P\left(\boldsymbol{n}^{0}\right) \Delta_{H}\left(\boldsymbol{n}^{0}\right)\right]_{H}$, where $P\left(\boldsymbol{n}^{0}\right)$ is the probability of a binary noise vector $\boldsymbol{n}^{0}$ being generated in the channel; and $\Delta_{H}\left(\boldsymbol{n}^{0}\right)$ denotes an indicator function that returns 1 when the posterior probability of a noise vector $\boldsymbol{n}$ given the syndrome $H \boldsymbol{n}^{0}(\bmod 2)$ is not maximized at $\boldsymbol{n}^{0}$, and 0 otherwise.

\section{Replicas in Gallager's Methodology}

Unfortunately, direct evaluation of the average error rate is generally difficult. Instead, Gallager's methodology upperbounds the average utilizing Chernoff's inequality [4]. In the current case, this approach provides a general bound

$$
\left[P_{E}\right]_{H} \leq\left[\sum_{\boldsymbol{n}^{0}} P\left(\boldsymbol{n}^{0}\right)\left(\sum_{\boldsymbol{n} \neq \mathbf{0}} \delta(H \boldsymbol{n}) \frac{P^{\lambda}\left(\boldsymbol{n} \oplus \boldsymbol{n}^{0}\right)}{P^{\lambda}\left(\boldsymbol{n}^{0}\right)}\right)^{\rho}\right]_{H},
$$

where $\delta(H \boldsymbol{n})$ becomes 1 when $H \boldsymbol{n}=0(\bmod 2)$ and vanishes otherwise; and $\oplus$ denotes the addition in modulo 2. Optimizing the parameters $0 \leq \lambda \leq 1$ and $\rho \geq 0$ makes the bound tighter. Evaluating Eq.(1) is still difficult as it involves a noninteger moment $\left[(\cdots)^{\rho}\right]_{H}$. A standard strategy in such cases is to further upperbound this expression by employing Jensen's inequality $\left[(\cdots)^{\rho}\right]_{H} \leq\left([\cdots]_{H}\right)^{\rho}$ with an additional constraint $\rho \in[0,1]$. As the bound is optimized by setting $\lambda=1 /(1+\rho)$ for a given $\rho$, we can obtain two types of bounds, depending

\author{
David Saad \\ Neural Computing Research Group, \\ Aston University, \\ Birmingham B4 7ET, UK
}

on whether Jensen's inequality is employed (i) after substituting $\lambda=1 /(1+\rho)$ or (ii) directly to Eq.(1). We shall refer to these strategies as J1 and J2, respectively. The replica method (RM), invented in statistical physics, offers another option for calculating the bound. This scheme evaluates Eq.(1) directly by analytically continuing the expression obtained for a natural number $\rho=1,2, \ldots$. for which analytical evaluation by the saddle point method becomes possible, to that of any real number $\rho$. Unfortunately, the validity of the replica method has not been proved in general, as well as that of the replica symmetry assumption used here [5]. Nevertheless, it can be shown that no known self-consistent condition is broken in the current case, which implies that the results obtained are likely to be correct [6]. For a BSC, characterized by a flip probability $0<p<1 / 2,\left[P_{E}\right]_{H}$ vanishes for $N \rightarrow \infty$ below a critical noise level $p_{t h}$ termed the zero error noise threshold [3, 7]. Lower bounds of $p_{t h}$, obtained by several methods and for various parameters, are compared in the Table below, where TP and SL denote the typical pairs analysis [3] and Shannon's limit, respectively. One can find that RM offers the most optimistic evaluation in all cases examined.

\begin{tabular}{|c|c|c|c|c|c|}
\hline$(j, k)$ & $\mathrm{J} 1$ & $\mathrm{~J} 2$ & $\mathrm{RM}$ & $\mathrm{TP}$ & $\mathrm{SL}$ \\
\hline$(3,6)$ & 0.0678 & 0.0915 & 0.0998 & 0.0915 & 0.109 \\
\hline$(4,6)$ & 0.1705 & 0.1709 & 0.173 & 0.1709 & 0.174 \\
\hline$(2,4)$ & 0 & 0.0286 & 0.0286 & 0.0286 & 0.109 \\
\hline
\end{tabular}

ACKNOWLEDGMENTS

Support by Grants-in-aid 13780208, JSPS (YK), The Royal Society and EPSRC-GR/N00562 (DS) is acknowledged.

\section{REFERENCES}

[1] R. G. Gallager, "Low-density Parity-check Codes," MIT Press, Cambridge, MA, 1963.

[2] D. J. C. MacKay, "Good Error-correcting Codes Based on Very Sparse Matrices," IEEE Trans. Infor. Theory, vol. 45, pp. 399431, 1999.

[3] S. Aji, H. Jin, A. Khandekar, D. J. C. MacKay, R. J. McEliece, "BSC Thresholds for Code Ensembles Based on "Typical Pairs" Decoding," In: Marcus, B., Rosenthal, J. (eds): "Codes, Systems and Graphical Models," Springer Verlag, New York, pp. 195-210, 2001.

[4] R. G. Gallager, "A simple derivation of the coding theorem and some application," IEEE Trans. Infor. Theory, vol. IT-11, pp. 3-18, 1965.

[5] H. Nishimori, "Statistical Physics of Spin Glasses and Information Processing," Oxford University Press, Oxford UK, 2001.

[6] Y. Kabashima, N. Sazuka, K. Nakamura and D. Saad, "Tighter decoding reliability bound for Gallager's error-correcting code," Phys. Rev. E, vol. 64, pp. 046113[1-4], 2001.

[7] G. Miller and D. Burshtein, "Bounds on the MaximumLikelihood Decoding Error Probability of Low-Density ParityCheck Codes," IEEE Trans. Infor. Theory, vol. 47, pp. 2696$2710,2001$. 